\title{
Integrated Dynamic Physiological Model for Drug Infusion Simulation Studies
}

\author{
M. Elisa Montain, Aníbal M. Blanco, and J. Alberto Bandoni* \\ Planta Piloto de Ingeniería Química, PLAPIQUI (UNS-CONICET) Camino La Carrindanga km. 7, 8000 Bahía Blanca, Argentina
}

Supporting Information

ABSTRACT: This work presents a dynamic model that integrates some of the main processes of the human physiology. It is aimed at simulating the behavior of most relevant variables in response to the administration of typical drugs used in medical procedures. The model integrates the following submodels available in the open literature: (i) cardiovascular, (ii) baroreflex, (iii) respiration, (iv) transport and distribution of substances, (v) pharmacological effects of drugs. The integrated model has a difficult mathematical structure due to the nonlinearity present in most relationships and the occurrence of time events associated with heart valves and other discontinuous phenomena. An efficient computational implementation was developed in the Fortran programming language and applied to several case studies. The results are in reasonable agreement with published experimental data although further studies are required to improve the model accuracy for real applications. The simulation model can be used as a computational tool for medical training and off-line diagnosis, as well as the basis for a patient specific online decision support system.

\section{INTRODUCTION}

Mathematical modeling in human physiology experimented a tremendous growth in recent decades. This type of research is currently referred as "in silico" by analogy with the more traditional "in vivo" and "in vitro" experimental studies in medical research. Large collaborative research initiatives, such as the ten-year-old project Physiome ${ }^{1}$ and the more recent ITFoM project ${ }^{2}$ of the European Community are among the many currently under development. These types of international multidisciplinary efforts give support to the assertion that mathematical modeling is becoming the new paradigm of medical research at the beginning of this century. ${ }^{3}$

Interdisciplinary collaboration among medicine, physics, mathematics, engineering, biochemistry, electronics, and biotechnology is giving a renewed impetus not only to the basic research but also to the development of computational tools for health caring. Different approaches, ranging from molecular to lumped parameter modeling passing through computational fluid dynamics, have been used in the literature to model the human physiology, covering from nanometers to meters in the spatial scale and from microseconds to the human lifetime in the temporal scale. ${ }^{1}$

A particularly challenging problem that is usually faced in care units, which could be considered using mathematical modeling, is the maintenance of the main physiological variables of the patients within acceptable ranges. Such control is in general achieved by means of the administration of drugs. The type of infused drug depends on the specific variable to be controlled. For example, arterial pressure and cardiac output are usually controlled using sodium nitroprusside and dopamine. In certain cases, it is also required to induce anesthesia by administrating appropriate agents, intravenously or via the respiratory system. Usually, physicians use their criterion to regulate the infusion rates and infer difficult to monitor variables, such as the depth of anesthesia, from indirect measurements.
Many mathematical models have been developed so far to represent the complex interactions in the physiology of human beings in order to assist in the decision making in the medical practice. From the pioneering work from Guyton and co-workers in the $1970 \mathrm{~s},{ }^{4}$ a large amount of contributions have been proposed to model different subprocesses of such a complex system. ${ }^{5}$ A comprehensive review of such work is beyond the scope of this article. The developed models have been used to study specific physiological issues, ${ }^{6-10,20}$ address the parameter estimation problem, ${ }^{11,12}$ and investigate the pharmacologic effect of drugs. ${ }^{13,14}$ Such models have been more recently used with control purposes. For example, strategies based on a model-based control approach were developed to assist in the hemodynamic and anesthetic regulation of patients. ${ }^{15-19}$

According to our knowledge, most models were developed to study specific physiologic subsystems or aimed at investigating particular processes (i.e., anesthesia, control strategies). The main purpose of this contribution is to integrate meaningful available models to develop a tool to be used from a process systems engineering approach to carry out in a systematic fashion, simulation, parameter estimation, dynamic optimization, and control studies.

In this work, a model that integrates different physiological processes is presented and used to simulate the response of the main physiological variables to the administration of different types of drugs. The overall model developed here comprehends the interconnection of the following submodels taken from the biomedical engineering research literature: (i) cardiovascular (circulatory) system, (ii) baroreflex, (iii) respiratory system,

\section{Special Issue: Jaime Cerdá Festschrift}

Received: February 28, 2014

Revised: July 31, 2014

Accepted: August 29, 2014

Published: August 29, 2014 
(iv) transport and distribution of substances, (v) pharmacologic effect of drugs. The model is essentially a differential-algebraic system of equations that presents "state events" due to the presence of the cardiac valves and other discontinuous processes. Such discontinuity confers to the model challenging features from an implementation point of view. The results are qualitatively compared to experimental data provided in the literature to perform a sort of validation.

The paper is organized as follows. Section 2 describes the different models of each subsystem and Section 3 details their integration and interrelations. Section 4 discusses the computational implementation and resolution issues. Section 5 presents the simulation case studies corresponding to different drug infusions together with a qualitative comparison with data gathered from the open literature. A conclusions and future work section closes the article. Appendixes 1 to 5 provide a detailed description of the mathematical models of the different subsystems and are available as electronic Supporting Information of this paper.

\section{SUBSYSTEMS DESCRIPTION}

In this section, the different submodels used in the paper are briefly introduced. For the sake of completeness, the detailed set of equations, variables and parameters of each model are provided in the appendices.

Cardiovascular System. The cardiovascular system model is taken from Ottesen et al. ${ }^{5}$ It is an overall lumped pulsatile model that captures the main features of the blood circulation system. Lumped cardiovascular models may be divided into two subclasses. The first class contains the nonpulsatile models where the heart is described by some variant of Starling's law. The second class contains the pulsatile models where the heart is typically guided by a classic time-varying elastance function. A lumped pulsatile model was selected in this contribution because it can predict realistic levels of flows and pressures along the cardiovascular system, thus it can be used to study different scenarios like hypertension and weak heart.

The adopted cardiovascular model represents the different organs by a series of interconnected elastic chambers (Figure 1), describing the pumping heart and vascular systems (systemic and pulmonary circulation).

The heart is made up of four elastic chambers: Left Atrium $(L A)$, Left Ventricle $(L V)$, Right Atrium $(R A)$, and Right Ventricle $(R V)$. The contractile properties of the ventricles are modeled by means of time varying elastances. Atria are modeled as passive chambers described by constant elastances. The heart possesses four valves that connect the different chambers: Mitral Valve $(M V)$, Aortic Valve $(A V)$, Tricuspid Valve $(T V)$, and Pulmonary Valve $(P V)$. Such valves allow the circulation of blood between the chambers if the pressure in the downstream chamber is lower than the pressure in the upstream chamber, otherwise, they remain closed. Regarding the vasculature, arteries are represented by three elastic chambers for the systemic and pulmonary sections $\left(A_{\mathrm{S} 1}, A_{\mathrm{S} 2}, A_{\mathrm{S} 3}, A_{\mathrm{P} 1}, A_{\mathrm{P} 2}, A_{\mathrm{P} 3}\right)$, whereas veins are lumped into two elastic chambers per section $\left(V_{\mathrm{S} 1}, V_{\mathrm{S} 2}, V_{\mathrm{P} 1}\right.$, $\left.V_{\mathrm{P} 2}\right)$. Each compartment represents a different section of the vascular tree. For example, chambers $A_{\mathrm{S} 3}$ and $V_{\mathrm{S} 1}$ correspond to the capillary sections in organs, whereas the capillary section at the pulmonary level is represented by chambers $A_{\mathrm{P} 3}$ and $V_{\mathrm{P} 1}$.

Each chamber is modeled through a mass balance. The volume of the chamber is the dynamic state. In the atria and ventricles, where the inertial effects are important, a momentum balance is also required. The flow rate is the corresponding state variable in

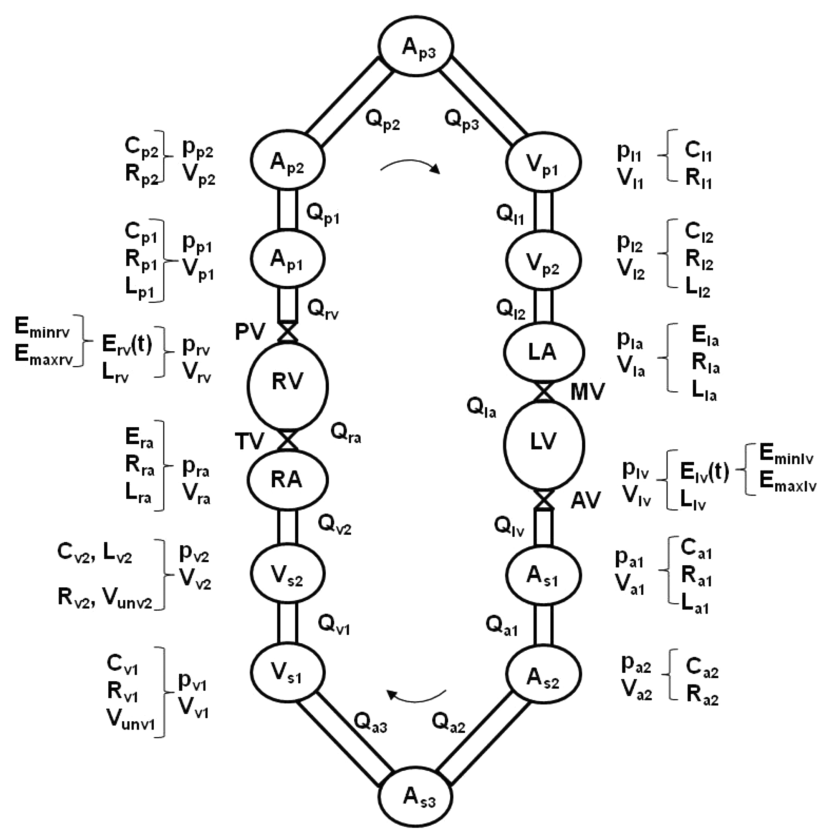

Figure 1. Cardiovascular system scheme. The model contains a systemic and pulmonary circuit and the heart. Circles represent elastic chambers to model specific sections of the vasculature.

such cases. For all chambers, a linear relationship between pressure and volume is adopted. In order to model the pumping effect of the ventricles, a sinusoidal driving function provides the time varying elastances of such chambers. The cardiac valves are represented as switches between two models depending on upstream and downstream pressures. The complete set of equations of the cardiovascular model, together with a description of its variables and parameters, are presented in Supporting Information Appendix 1.

Baroreflex System. The baroreceptors are sensors which monitor changes in Mean Arterial Pressure (MAP) and actuate as measurement devices in a control loop to provide short-term pressure regulation. Based on the sensor signal $(n)$, the central nervous system generates a control action $(n s, n p)$ to manipulate certain elements of the cardiovascular system in order to restore the original pressure (heart rate, heart elastance, vein compliences, etc.). Figure 2 shows the baroreceptor system scheme, its inputs and outputs and the interconnection with the cardiovascular system. The adopted model of the baroreflex, taken from Ottesen et al., ${ }^{5}$ is reproduced in detail in Supporting Information Appendix 2.

Respiratory System. This submodel represents the transport of gaseous components from the atmosphere, or a respiratory mask, to the alveoli, where matter exchange with blood takes place. The states of the model are the pressures in the different sections of the airways from the nasal and oral cavities to the alveolar sacs, together with the molar fractions of oxygen, carbon dioxide and, eventually, inhalable anesthetic agents. The pressure generated by the thorax muscles is represented by a sinusoidal law $\left(U_{t}\right)$, where frequency and amplitude are chosen in order to mimic the typical ventilation function. If present, the ventilation mask is also represented by an appropriate sinusoidal function $\left(U_{\mathrm{m}}\right)$. The model, taken from Christiansen and Dræby, ${ }^{21}$ is reproduced in Supporting Information Appendix 3 and schematically depicted in Figure 3.

Diffusion was neglected in the current version of the model because it is considered that natural or forced ventilation takes 


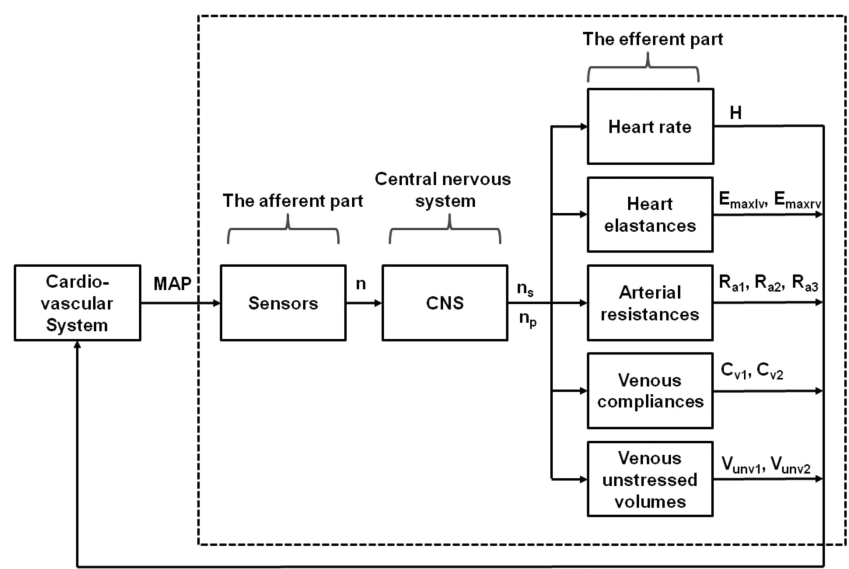

Figure 2. Baroreflex scheme coupled to the cardiovascular model. The mean arterial pressure MAP is used by the barorreceptor model to control heart rate $H$ and the cardiac contractility $E_{\text {maxly }}, E_{\text {maxrv }}$. Also, it modifies the three systemic arterial resistances $R_{\mathrm{a} 1}, R_{\mathrm{a} 2}$, and $R_{\mathrm{a} 3}$; the systemic venous compliances $C_{\mathrm{v} 1}, C_{\mathrm{v} 2}$; and the systemic venous unstressed volumes $V_{\text {unv1 }}$ and $V_{\text {unv2 }}$.

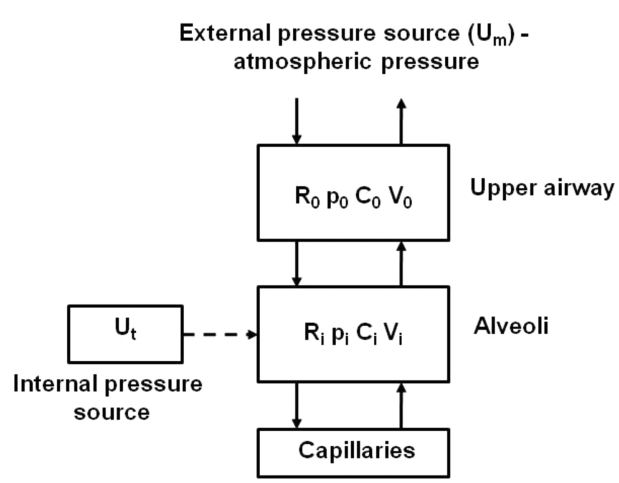

Figure 3. Respiratory system scheme. The chambers represent the upper airway and the alveoli. The model simulates artificial ventilation through the generator $U_{m}$ and the generator $U_{t}$ represents the natural ventilation.

place within the airways and therefore, laminar flow dominates over diffusive flow. ${ }^{5,21}$ Moreover, such hypothesis is also valid even under long periods without breathing.

Transport and Distribution System. Oxygen, carbon dioxide, and inhalable drugs get into the body via the respiratory system. Other drugs are usually intravenously administrated. In order to study the distribution of the different substances within the organs, the body is divided into several compartments ${ }^{21}$ as shown in Figure 4.

After exchanging oxygen and carbon dioxide in the lungs, blood distributes from the arterial pool toward the different organs. In each organ, a specific metabolism takes place and carbon dioxide is produced at the expense of oxygen consumption. The carbon dioxide enriched blood is collected in various venous pools and pumped toward the lungs, closing the loop. This system is connected with the cardiovascular model through the cardiac output $\left(Q_{\mathrm{a} 3}, Q_{\mathrm{p} 3}\right)$.

Each organ is modeled as part tissue $\left(V_{\mathrm{t}}\right)$, part blood $\left(V_{\mathrm{b}}\right)$ (see Figure 5) as proposed in Christiansen and Dræby. ${ }^{21}$ Carbon dioxide is produced at a constant rate, whereas oxygen reacts at a rate that depends on its concentration in the blood. The different drugs in the system are consumed at specific organs at rates depending on their concentration in blood. Mass balances model the concentration profiles of each component.

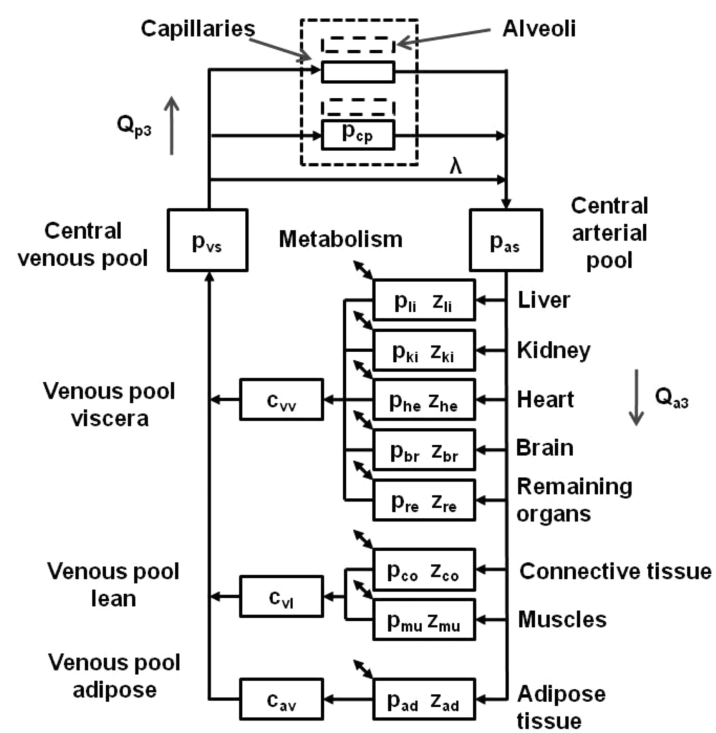

Figure 4. Blood distribution scheme. The model splits the body into $(13+n$-capillaries $)$ compartments, to represent main organs and tissues, capillaries, and blood pools.

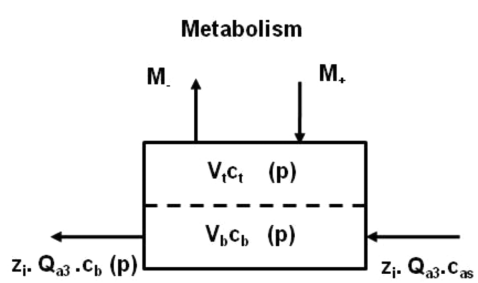

Figure 5. Scheme and expressions for the gas content of a general organ of the Transport and Distribution System.

The concentrations of oxygen, carbon dioxide and drugs in the blood and in the tissue of each organ are provided by the corresponding dissociation functions. ${ }^{22}$ The dissociation functions relate the tensions of the substances in the blood or tissue with their concentrations. The dissociation functions of oxygen and carbon dioxide are complex nonlinear relationships that depend on the blood $\mathrm{pH}$. On the other hand, linear relations can usually be assumed for drugs.

Finally, at the lung capillaries and alveoli (Figure 6), oxygen, carbon dioxide, and inhalable drugs are exchanged. This process is modeled through the mass balances of the different components.

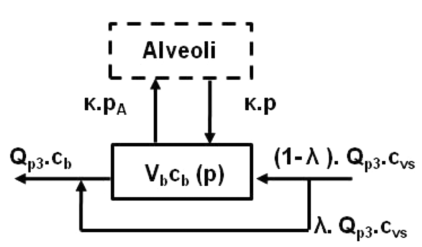

Figure 6. Scheme and expressions for the gas content in pulmonary capillary compartment of the Transport and Distribution System. The connection with the respiratory system is represented by dashed lines.

The model of the transport and distribution of substances, as presented in Christiansen and Dræby, ${ }^{21}$ is reported in Supporting Information Appendix 4.

Pharmacodynamic System. Drugs are administrated to patients, typically intravenously, with different purposes. 
For patients that are about to be operated on, anesthesia has to be induced by infusion of anesthetic agents such as thiopental and propofol. Other anesthetic agents, for example, isoflurane, can be also administrated via the respiratory system.

In order to monitor the level of hypnosis, the effect of the anesthetic agent on the bispectral index (BIS) is adopted. BIS is a measure of the effects of anesthetics and sedatives on the brain. It is based on the bispectral analysis of the electroencephalogram (EEG) of the patient. BIS is an indirect measure of the depth of anesthesia, as for the moment, there is not a direct one. BIS values range between 0 and 100, where 100 is characteristic of an awake patient EEG, whereas 0 denotes an isoelectric EEG signal. $B I S$ predicts with good accuracy the return to the state of consciousness, and is the only measure clinically accepted to monitor hypnosis. Several authors have studied the action mechanisms of the classic anesthetic agents propofol and isoflurane and their impact on the BIS measurement. ${ }^{24-32}$ In this work, the corresponding pharmacodynamics were modeled as proposed in Gentilini et al. ${ }^{16}$ and Ionescu et al. ${ }^{23}$

Other drugs (sodium nitroprusside, dopamine, etc.) have a specific effect on important physiological variables such as the mean arterial pressure and cardiac output. These variables are controlled in patients under surgery by careful infusion of such medicaments. From a modeling point of view, specific parameters of the cardiovascular system, such as elastances and resistances, are dynamically modified depending on the concentration of the drug in certain organs. The effect of the drugs on the physiological variables (Eff) adds up to the output of the baroreflex system as depicted in Figure 7. In Supporting Information Appendix 5, the equations for the pharmacodynamic effects of the drugs used in this study are provided. ${ }^{13,14,16,23}$

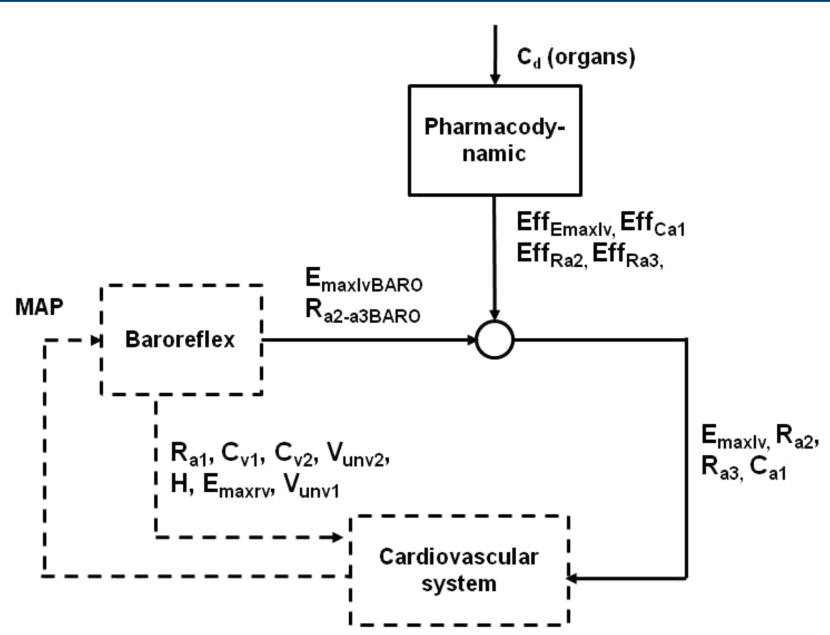

Figure 7. Drug pharmacodynamic scheme and its relationship with the Baroreflex and the Cardiovascular systems, detailed in dashed lines.

\section{INTEGRATED MODEL}

All the submodels described in the previous section were integrated in a single framework in order to dynamically simulate some important human physiological processes. In Figure 8, the interconnection between the submodels is depicted. The variable that connects the cardiovascular subsystem with the transport subsystem is basically the heart flow rate (cardiac output), which at the pulmonary section is represented by the flow rate $Q_{p 3}$ and at the systemic level by the flow rate $Q_{33}$. Each organ is fed with a fraction of blood flow taken from $Q_{\mathrm{a} 3}$. The transport subsystem is in turn connected to the respiratory subsystem by means of the partial pressures of the gaseous components, which are exchanged at the pulmonary capillaries and the alveoli $\left(P_{\mathrm{CO}_{2}}, P_{\mathrm{O}_{2}}, P_{\mathrm{D}}\right)$.

If the drug is administrated via the respiratory system, it is modeled as a gaseous component that reaches the transport and distribution subsystem through the alveoli-capillaries interphase in the lungs. Drugs infused intravenously, $C_{\mathrm{d}}$ (injectable), or via the respiratory system, $C_{\mathrm{d}}$ (inhalable), distribute according to particular kinetics among the different organs through the transport system, where the concentration of the drug, $C_{\mathrm{d}}$ (organs), evolves according to the corresponding mass balance. If the drug has a hemodynamic effect, its concentration in the arterial blood pool modifies certain parameters of the cardiovascular system $\left(E f f_{\text {Emaxlv }}, E f f_{\mathrm{Ra} 2}, E f f_{\mathrm{Ra} 3}, E f f_{\mathrm{Ca1}}\right)$, which are quantified through the phamacodynamic system and typically impacts on the MAP and on the cardiac output introducing a feedback loop (Loop A). Moreover, because MAP is monitored by the baroreflex, which also actuates on some of the cardiovascular parameters that are influenced by the drugs $\left(E_{\text {maxlvBAROO }}, R_{\mathrm{a} 2-\mathrm{a} 3 \mathrm{BARO}}\right)$, a second feedback loop is established (Loop B). The effect of the drugs and the changes introduced by the baroreflex actuate on parameters $E_{\text {maxlv }}, R_{\mathrm{a} 2}, R_{\mathrm{a} 3}, C_{\mathrm{a} 1}$, which are fed to the cardiovascular system, together with those parameters that are only modified by the baroreflex system $\left(R_{\mathrm{a} 1}, H, E_{\text {maxrv }}, C_{\mathrm{v} 1}, C_{\mathrm{v} 2}, V_{\text {unv1 } 1}, V_{\text {unv2 }}\right)$, establishing Loop $C$. If the drug has an anesthetic effect, its concentration in a specific compartment can be used to quantify the depth of anesthesia through the bispectral index (BIS).

Overall, the simulation model is basically a system of differential equations which is numerically integrated from a given initial condition for each state variable (see Supporting Information). A small set of nonlinear algebraic equations is solved at the beginning of each heart-beat to obtain the carbon dioxide concentration in blood, since it cannot be explicitly calculated due to its complex dissociation function. Additional variables that are explicit functions of the state variables (MAP, $C O, B I S$, etc.) are straightforwardly calculated along the integration process. In the following section, implementation details regarding the numerical integration of the equations system are provided.

\section{IMPLEMENTATION ISSUES}

From a mathematical point of view, the described model has several challenging properties, being the most immediate significant nonlinearity present in almost every equation. However, in our opinion, the most complicating issue is the discontinuity introduced by the cardiac valves (see Supporting Information Appendix 1) and the unidirectional events that take place in the respiration (see Supporting Information Appendix 3). These discontinuities give rise to the so-called "state events", which confer a hybrid nature (continuous/ discrete) to the model. A state event is "a change" in the model equations triggered by some state variables reaching a specific condition. For example, a cardiac valve is open when the upstream pressure is greater than the downstream pressure. In this situation, the corresponding flow rate is quantified by the momentum balance. When the downstream pressure is larger than the pressure in the chamber, the valve closes and the flow rate is set to zero. This phenomenon is particularly intense in the integrated model (it occurs approximately every $0.8 \mathrm{~s}$ ) due to the sinusoidal nature of the driver functions of the cardiovascular and the respiratory systems. ${ }^{5}$ 


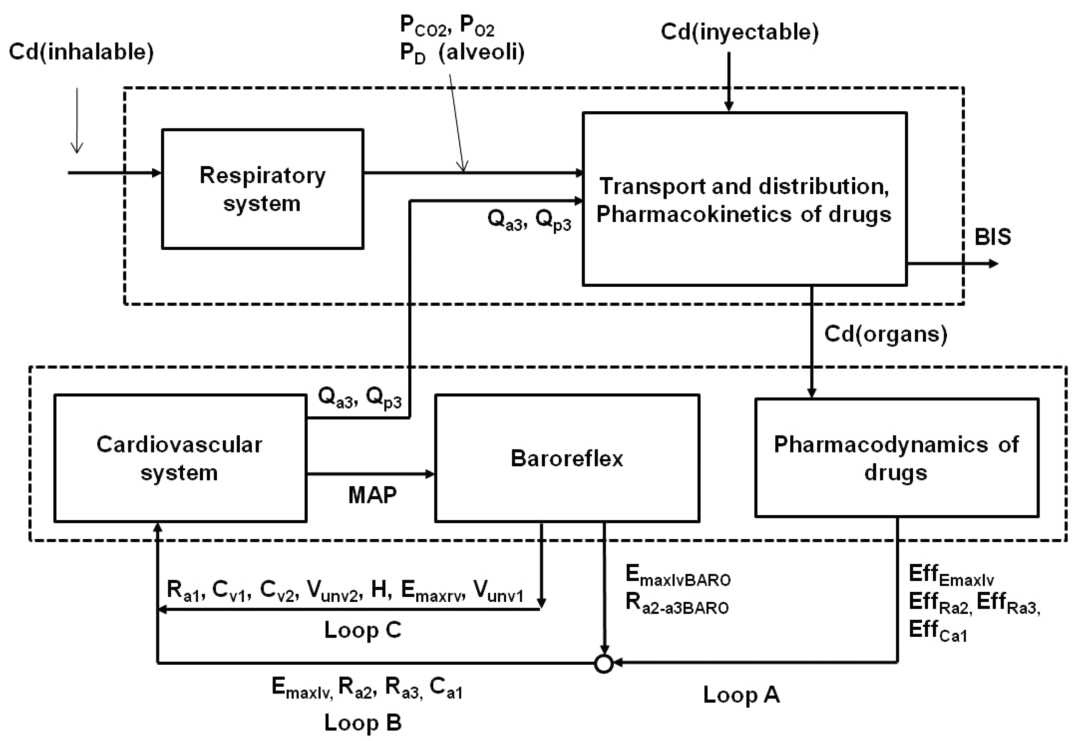

Figure 8. Submodels integration and flow of information between systems.

Hybrid dynamic models and state event handling have been largely studied in the process systems engineering discipline due to the inherent discontinuous nature of chemical process operations. ${ }^{33-37}$ Most of the techniques for state event handling are based on variations of the "discontinuity locking" approach. Conceptually, this strategy requires the simulation of the current model until a state event is detected (event detection). Then, more or less sophisticated techniques are implemented in order to identify the exact moment of the event occurrence (event location). Finally, the new model is further integrated from the identified instant. The appropriate handling of time events is required for at least two reasons: (i) if the precise moment of the event is not adequately identified, a significant error can be associated with the initial condition for the integration of the next model, which accumulate along the integration horizon, and (ii) in the case that several events take place in a very short period, to exactly locate the first one is a must because dramatically misleading responses that can be obtained otherwise.

Despite its importance, the hybrid nature of the cardiovascular model has received very little attention in the physiological modeling literature. According to our knowledge, only Smith ${ }^{9}$ explicitly recognized the issue and adopted the "events" function in the Matlab implementation to solve the hybrid differential equation model of the cardiovascular system. It is also mentioned in that contribution that a simulation of 100 heart-beats may demand about $10 \mathrm{~min}$ in certain cases, which is clearly unacceptable for many applications. Finally, the author claims that the use of optimized code and a faster programming language might improve those times significantly.

In Olufsen et al.," the "state event" issue is addressed using a variable resistance for the valves, where a high value of the resistance simulates the closure of the valve.

In the proposed integrated model, it was observed that the use of sophisticated schemes to deal with state events introduce a significant computational burden, making even short-term simulations unpractical for intense experimentation. Therefore, an efficient implementation of the model is required.

There is also little detail on the programming aspects of the different models presented in the literature. In Christiansen and Dræby, ${ }^{21}$ a Runge-Kutta-Felhberg formula is adopted for the integration of the respiratory-transport system of differential equations in a C++ implementation. In Smith, ${ }^{9}$ a Matlab integration routine based on numerical differentiation formulas well adapted to stiff models is used.

In our contribution, an explicit Euler formula is adopted. Explicit Euler is the simplest integration scheme. Although it is easy to implement and provides the fastest responses, it is known to present limited efficiency with stiff problems, where implicit schemes are the most appropriate. Moreover, it is well known that the stability and accuracy of explicit algorithms is highly dependent on the adopted integration step. Very small integration steps ensure stability and accuracy at the expense of large computation times.

According to our computational experience on this particular model, explicit Euler with $\Delta t=0.0005 \mathrm{~s}$ presents an adequate balance among computation speed, stability, and accuracy, avoiding therefore the use of implicit schemes whose computational burden, stemming from the resolution of nonlinear systems of algebraic equations, is significant. ${ }^{39}$

Moreover, explicit Euler with a small integration step provides a simple way of handling state events. During the integration, state conditions are checked in each step. Once an event is detected, a switch to the appropriate model is immediately performed, without delaying the integration process with extra calculations.

Finally, the model was implemented in Fortran code to generate an efficient program and to have at disposal a large number of mathematical functions and subroutines for intermediate calculations. A simulation period of $80 \mathrm{~min}$ can be performed in $1 \mathrm{~min}$ and $40 \mathrm{~s}$ of CPU time, with the actual implementation (processor INTEL CORE i5-2500 $3.3 \mathrm{GHz}, 4$ GB RAM). Additionally, an adequate Excel interface was developed for easy data exchange and results visualization.

\section{MODEL VALIDATION}

The validation of a model is aimed to identify the level of agreement between the predictions of the adopted mathematical expressions and the actual behavior of the system under study. In practice, validation is reduced to calculate how approximate are the model output variables to the measured variables of the real process. ${ }^{40}$ The comparison of measured and simulated data can be performed qualitatively or quantitatively. 
Table 1. Drugs, Doses, and Their Main Effects on the Cardiovascular System

\begin{tabular}{|c|c|c|c|}
\hline drug & variable modified & effect & dose \\
\hline $\begin{array}{l}\text { sodium nitroprusside } \\
\text { (SNP) }\end{array}$ & resistance of systemic arteries & decreasing $M A P$ & \\
\hline increasing $\mathrm{CO}$ & $1 \mu \mathrm{g} / \mathrm{kg} / \mathrm{min}$ & & \\
\hline dopamine (DP) & $\begin{array}{l}\text { resistance of systemic arteries, maximum } \\
\text { systolic elastance }\end{array}$ & increasing $M A P$, increasing $C O$ & $5 \mu \mathrm{g} / \mathrm{kg} / \mathrm{min}$ \\
\hline propofol (PFL) & arteries distensibility, BIS (bispectral index) & decreasing $M A P$, unconsciousness & $\begin{array}{l}\text { induction: } 225 \mu \mathrm{g} / \mathrm{kg} / \mathrm{min} \text { for } 5 \mathrm{~min} \text {, } \\
\text { maintenance: } 80 \mu \mathrm{g} / \mathrm{kg} / \mathrm{min} \text { average }\end{array}$ \\
\hline
\end{tabular}

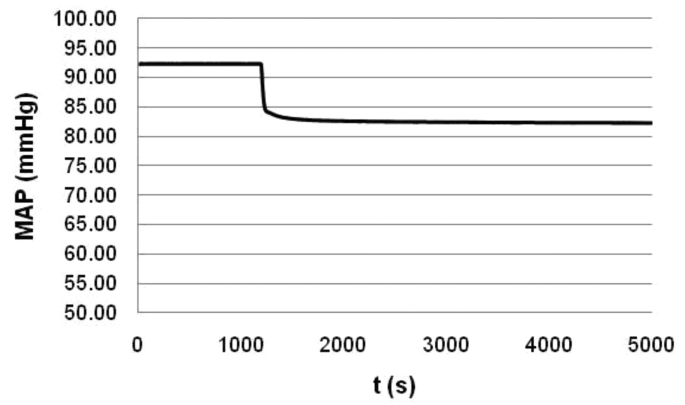

a. MAP

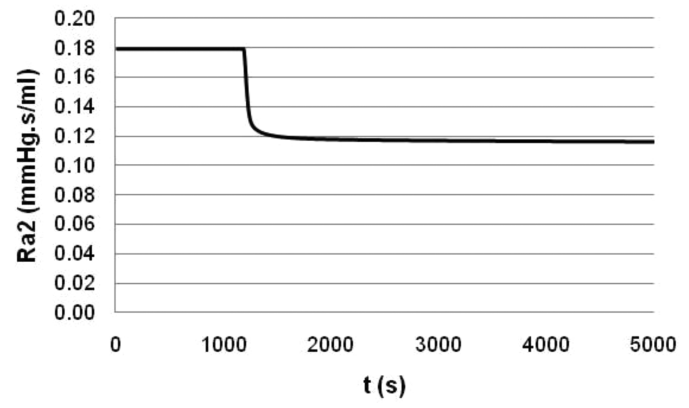

c. $R_{a 2}$

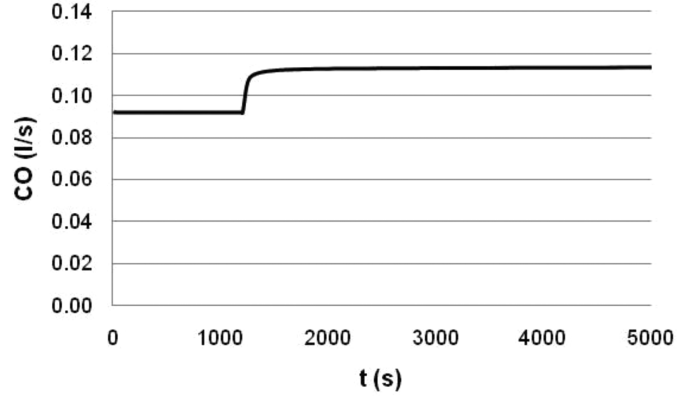

b. $\mathrm{CO}$

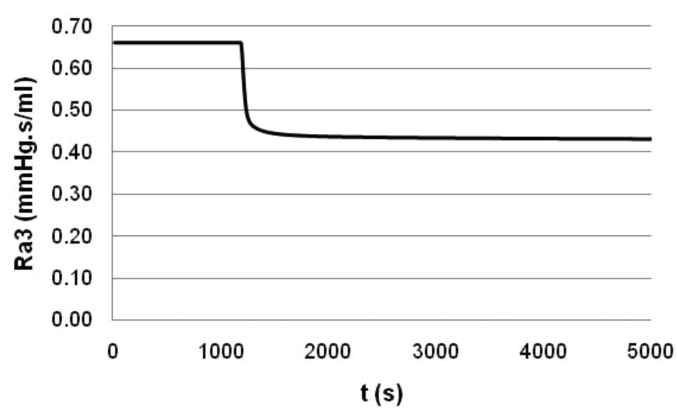

d. $R_{a 3}$

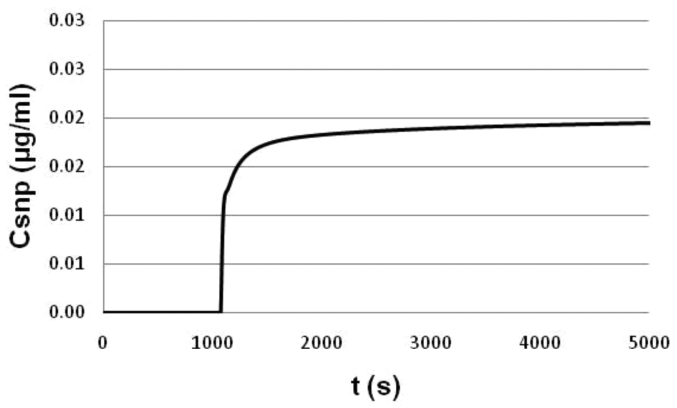

e. $C_{s n p}$

Figure 9. Case I: Variables' time profiles predicted by the model under the effects of the administration of $1 \mu \mathrm{g} / \mathrm{kg} / \mathrm{min}$ of SNP. (a) MAP; (b) CO; (c) $R_{\mathrm{a} 2} ;(\mathrm{d}) R_{\mathrm{a} 3} ;(\mathrm{e}) C_{\mathrm{SNP}}$.

Qualitative approaches involve analyzing plots of the model and the corresponding process variables to visually identify differences. Quantitative methods are based on performance measures such as the root-mean-square error, the Theils inequality coefficient and the relative error among others. The closer to zero the value of these performance measures, the better the model prediction. In this work, a qualitative approach is adopted.

The validation of physiological dynamic models is particularly difficult, both for the large number of parameters and variables and the difficulties of obtaining them in practice. Therefore, despite the model presented in this paper provides dynamic profiles for more 


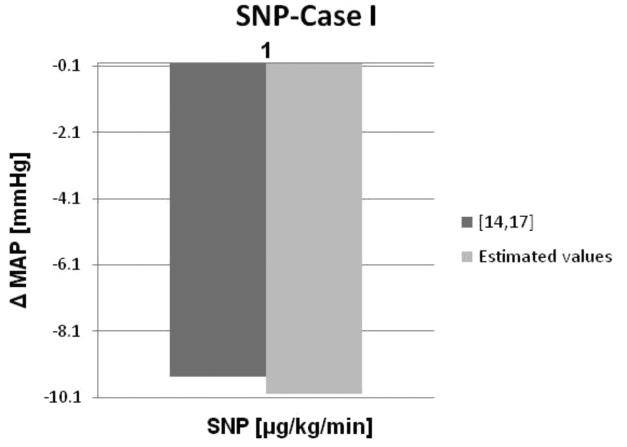

a. $M A P$

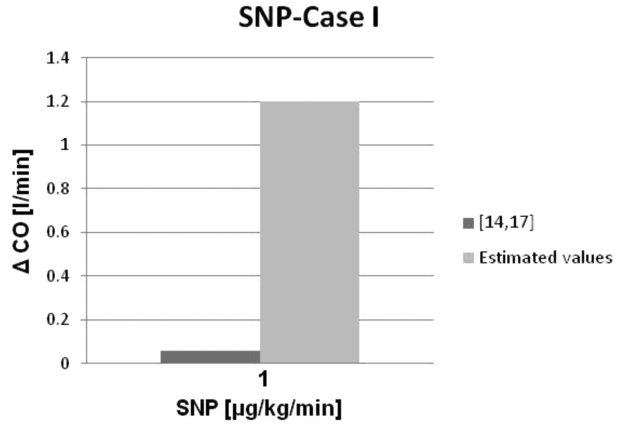

b. $C O$

Figure 10. Case I: Difference between initial and final variables states predicted by the model (light bars) versus data reported in the references Gopinath et al. ${ }^{14}$ and Dua and Pistikopoulos ${ }^{17}$ (dark bars), when using $1 \mu \mathrm{g} / \mathrm{kg} / \mathrm{min}$ of SNP drug. (a) MAP; (b) CO.

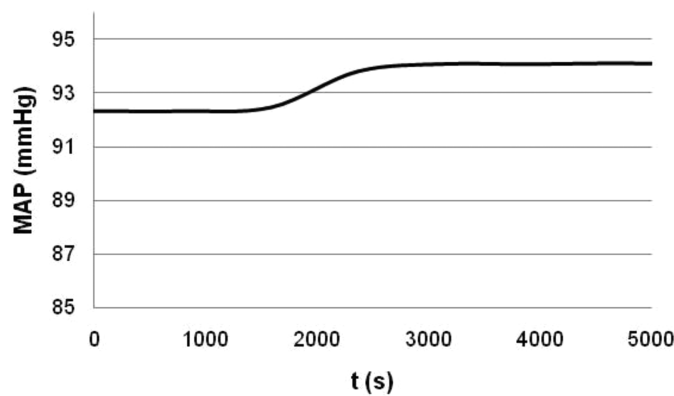

a. $M A P$

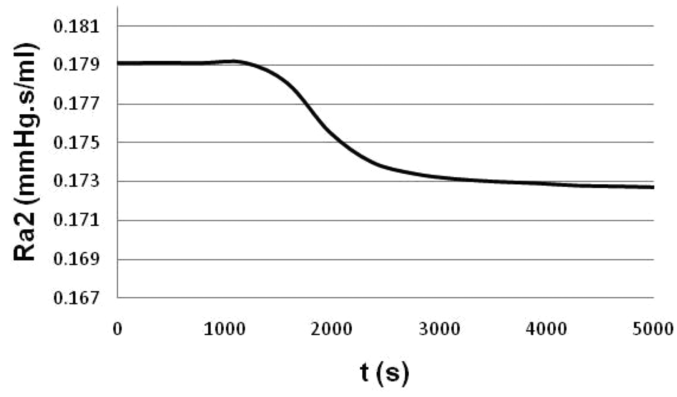

c. $R_{a 2}$

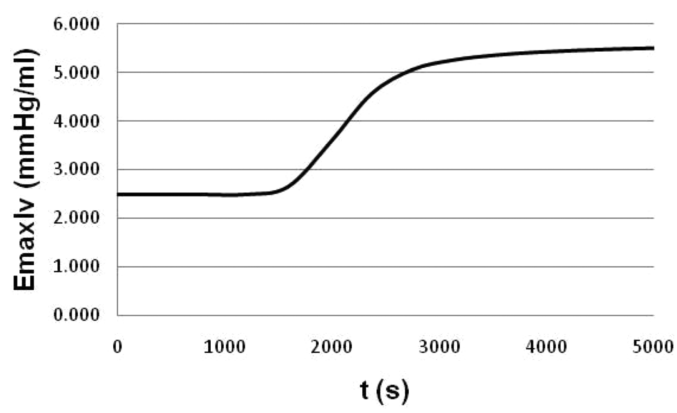

e. $E_{\max l v}$

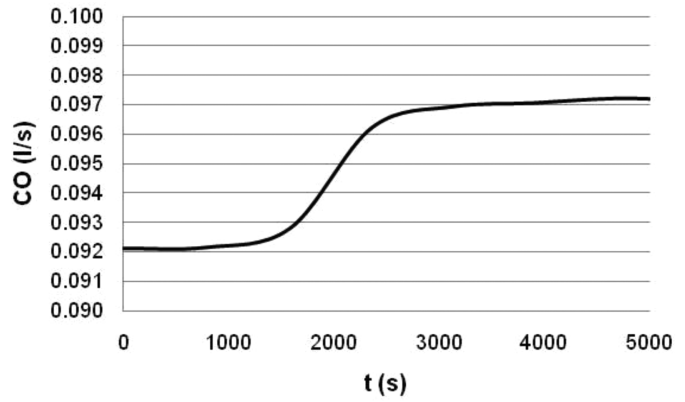

b. $C O$

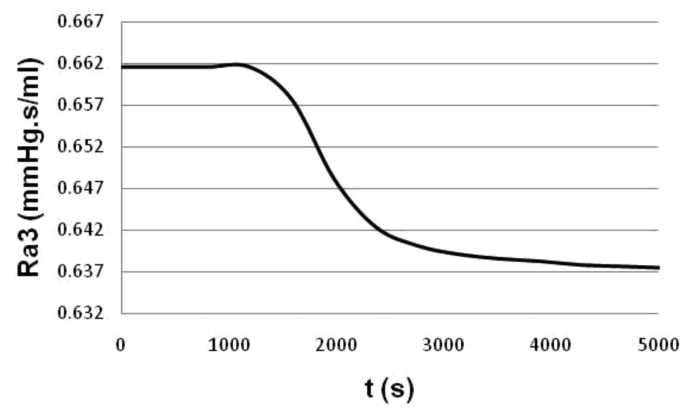

d. $R_{a 3}$

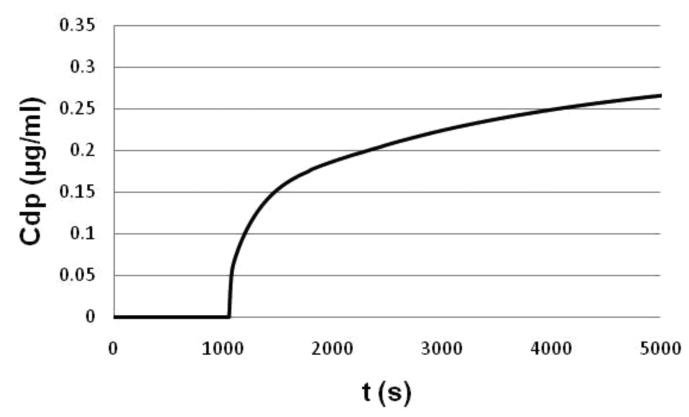

f. $\boldsymbol{C}_{d p}$

Figure 11. Case II: Variables time profiles predicted by the model under the effects of the administration of $5 \mu \mathrm{g} / \mathrm{kg} / \mathrm{min}$ of DP. (a) MAP; (b) CO; (c) $R_{\mathrm{a} 2} ;(\mathrm{d}) R_{\mathrm{a} 3} ;(\mathrm{e}) E_{\text {maxlv }} ;(\mathrm{f}) C_{\mathrm{dp}}$.

than one hundred variables, we choose to test only a subset of critical variables to gain the big picture of the model behavior, using parameters and variables profiles for a "healthy" individual reported in the medical and engineering research literature.
To validate the model presented in this paper, several simulation studies considering independent infusion of anesthetic and other drugs were conducted. Then, a qualitative validation of some important model variables was performed by 


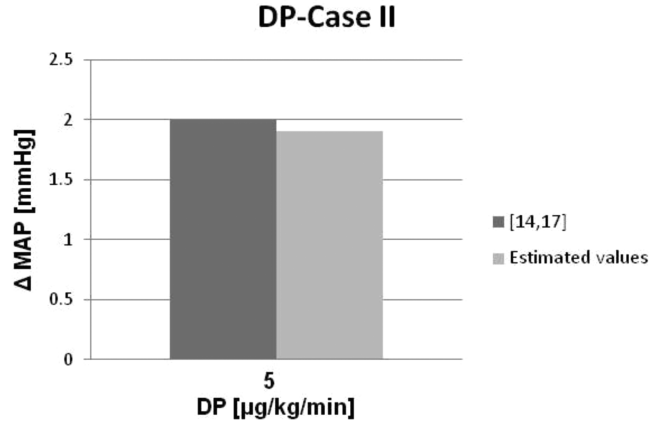

a. $M A P$

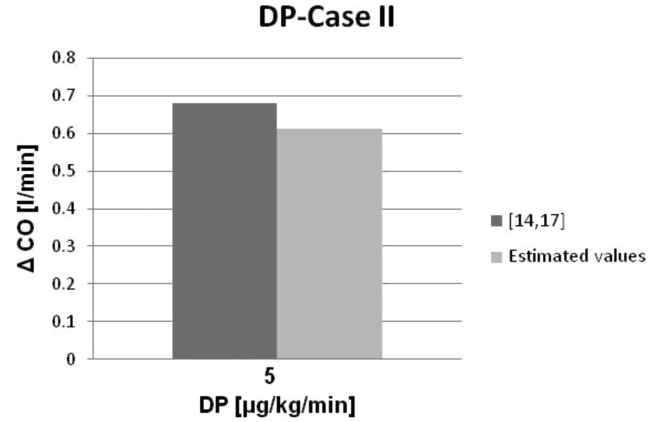

b. $C O$

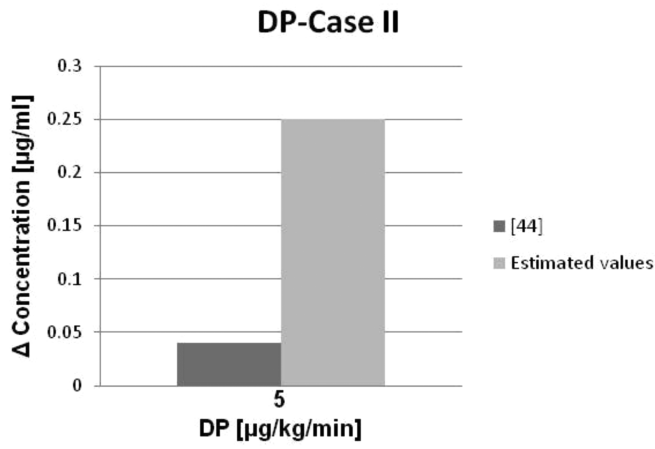

\section{c. DP Concentration}

Figure 12. Case II: Difference between initial and final variables states predicted by the model (light bars) versus data reported in the literatures (dark bars) when using $5 \mu \mathrm{g} / \mathrm{kg} / \mathrm{min}$ of DP drug. (a) MAP, (b) CO [data from Gopinath et al. ${ }^{14}$ and Dua and Pistikopoulos ${ }^{17}$, (c) DP concentration [data from MacGregor et al. ${ }^{44}$.

comparing the differences between their initial and final states (after the administration of the drug) predicted by the model, with these values reported in the literature.

Two different groups of data from the literature were used in the validation process: (i) clinical studies where hemodynamic and anesthetic variables are monitored for several patients ${ }^{41-47}$ and (ii) "in silico" studies which simulate the human behavior under the administration of dopamine, sodium nitroprusside, propofol, and isoflurane. ${ }^{15,17,23}$

In all cases, the simulations are initialized from the steady state resulting from the adopted parametrization, which corresponds to an average individual, meaning that it does not represent a specific individual or group. The comparisons are oriented, therefore, to estimate the tendencies and the magnitudes of the model response compared with those reported in the clinical studies (or from validated models) but not to provide an accurate prediction of the experimental evidence because the initial condition of the experiment and its specific parametrization are unknown.

In Table 1, the description of the drugs used in the case studies, together with the applied doses, are detailed. In all cases, a step change of the indicated drug starts after 15 min of steady state simulation and is administrated during $70 \mathrm{~min}$.

Case I: Sodium Nitroprusside (SNP) Administration. SNP is a potent vasodilator, which is applied to provoke hypotension during surgery. Figure 9 shows the results of the administration of a $1 \mu \mathrm{g} / \mathrm{kg} / \mathrm{min}$ dose. SNP relaxes the soft muscles of arteries and veins allowing a rapid decrease of MAP (Figure 9a) as simulated in Dua and Pistikopoulos, ${ }^{17}$ while inducing a light increase in $\mathrm{CO}$ as shown in Figure $9 \mathrm{~b}$.
This behavior is identified in Gopinath. ${ }^{14}$ The effect of the SNP on the resistances of the systemic arteries is observed in Figure $9 \mathrm{c}$ and $\mathrm{d}$, which is in agreement with those reported in Gopinath et al. ${ }^{14}$ and Rao et al. ${ }^{15}$ The evolution of the concentration of SNP in the arterial blood is shown in Figure 9e.

The qualitative validation for the infusion of SNP is shown in Figure 10. The variables compared against experimental data taken from Gopinath et al. ${ }^{14}$ and Dua and Pistikopoulos ${ }^{17}$ are $M A P$ and $C O$.

From Figure 10a and b, it can be seen that the integrated model predicts correctly the tendency of the variations in MAP and $C O$ when $1 \mu \mathrm{g} / \mathrm{kg} / \mathrm{min}$ of SNP is administered, with a good quantitative agreement for $M A P$ but with a large discrepancy in $\mathrm{CO}$, as compared with experimentally observed data reported in Gopinath et al. ${ }^{14}$ and Rao et al. ${ }^{15}$

Case II: Dopamine (DP) Administration. DP is used in patients with hypotension, systemic vasodilatation or low cardiac output because it causes an increase in MAP and $C O{ }^{14}$ Figure $11 \mathrm{a}$ and $\mathrm{b}$ show the profiles of such variables, respectively. Figure $11 \mathrm{c}$ and $\mathrm{d}$ shows the action of DP on the resistance of the systemic arteries, ${ }^{14,15,17,41}$ whereas Figure 11e depicts the cardiac contractility. ${ }^{14}$ Figure $11 \mathrm{f}$ shows the concentration profile of DP in the arterial blood, which can be contrasted with the responses shown in MacGregor et al. ${ }^{44}$

The variables compared against experimental data for a qualitative validation are $M A P, C O$, and the concentration of DP in systemic arteries (Figure 12).

For MAP and CO, a good approximation is observed between the simulated and the experimental results (Figure 12a and b). In contrast, there is a significant overestimation of the model 


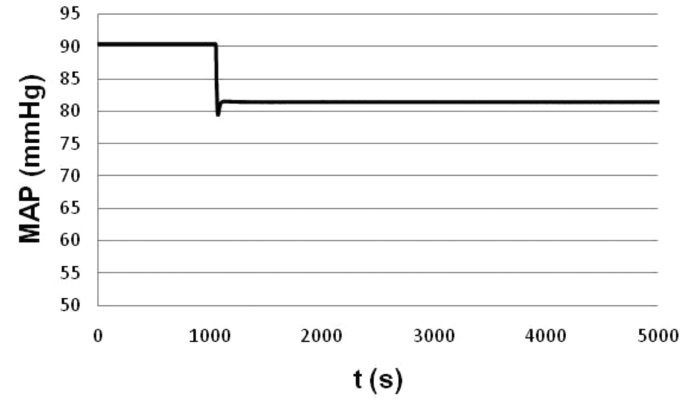

a. $M A P$

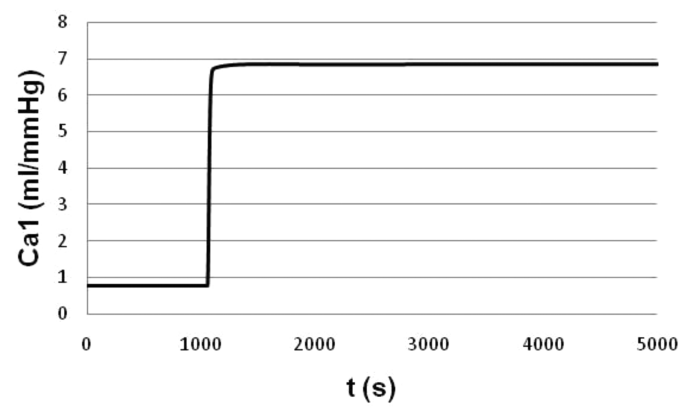

c. $C_{a 1}$

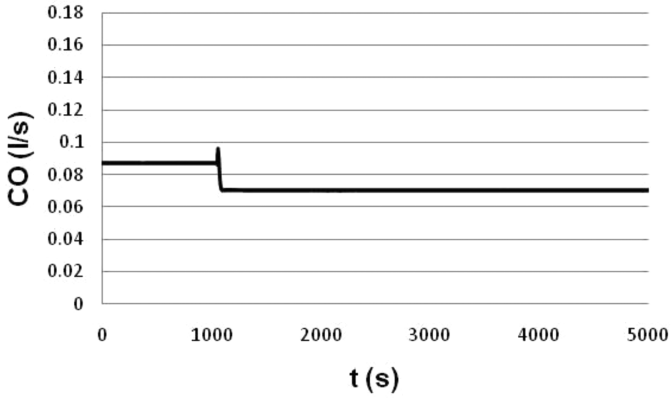

b. $C O$

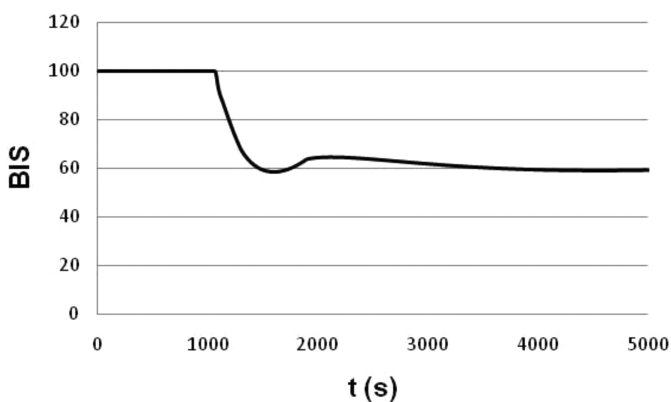

d. $B I S$

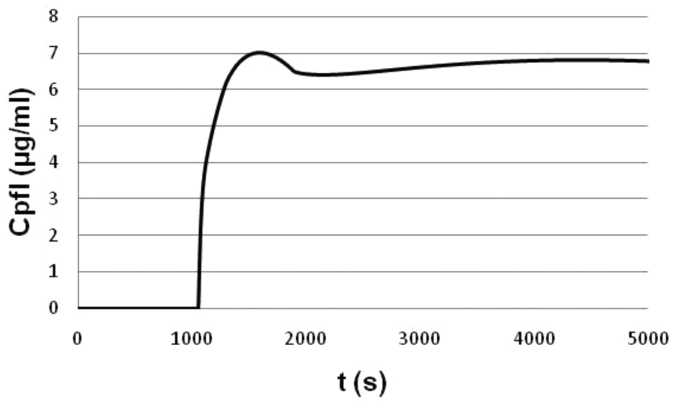

e. $C_{p f l}$

Figure 13. Case III: Variables time profiles predicted by the model under the effects of an induction of PFL at a rate of $225 \mu \mathrm{g} / \mathrm{kg} / \mathrm{min}$ during $5 \mathrm{~min}$, followed by an average infusion rate of the same drug at a rate of $80 \mu \mathrm{g} / \mathrm{kg} / \mathrm{min}$. (a) $M A P$; (b) $C O$; (c) $C_{\mathrm{a} 1}$; (d) BIS; (e) $C_{\mathrm{PFL}}$.

prediction with respect to the concentration of the drug in systemic arteries as reported in MacGregor et al. ${ }^{44}$ (Figure 12c).

Case III: Intravenous Propofol (PFL) Administration. Propofol is used to induce anesthesia (Figure 13). Figure 13d shows the evolution of the bispectral index to monitor the depth of anesthesia. This information can be contrasted with the results found in Ionescu et al. ${ }^{23}$ and Sreenivas et al. ${ }^{18} \mathrm{PFL}$ also produces an increase in the distensibility of the systemic arteries ${ }^{15,42}$ as shown in Figure 13c, causing a decrease in MAP and CO (Figure 13a and $\mathrm{b}$ respectively). Figure $13 \mathrm{e}$ shows the arterial blood concentration of PFL. ${ }^{45,46}$

For the PFL drug, a correct trend is observed in all variables in agreement with the experimental evidence (Figure 14). Regarding the magnitude of change, the model approximates well to the experimental data for BIS, whereas for the rest of the variables, rather large differences are observed.

Case IV: Inhalable Isoflurane (ISO) Administration. In Case IV, Isoflurane (ISO) is used to induce anesthesia (Figure 15). In Figure 15a, b, and c it is shown the influence of MAC (minimum alveolar concentration) over MAP, cardiac index, and systemic resistance, respectively. These results can be compared to those found in Malan et al. ${ }^{43}$ MAC is the concentration of isoflurane in the lungs that is needed to prevent movement (motor response) in response to surgical stimulus (pain). It was established that $M A C=1$ represents an isoflurane alveolar concentration of $1.15 \%{ }^{43}$ The values of the cardiovascular variables shown in Figure $15 \mathrm{a}, \mathrm{b}$, and $\mathrm{c}$ are the states reached after the corresponding $M A C$ has been maintained fixed for $20 \mathrm{~min}$.

As can be seen from Figure 15a and b, MAP decreases with any anesthetic concentration, whereas cardiac index ( $\mathrm{CO}$ divided by body surface area) increases. These responses are in agreement with the vasodilatation of the systemic arteries caused by isoflurane, which results in a decrease in the systemic resistances, Figure $15 \mathrm{c}$ (the systemic resistance is the sum of $R_{\mathrm{a} 1}, R_{\mathrm{a} 2}, R_{\mathrm{a} 3}, R_{\mathrm{v} 1}$ and $R_{\mathrm{v} 2}$ in Figure 1.). Figure $15 \mathrm{~d}$ shows the evolution of the bispectral index to monitor the depth of anesthesia in time, for a $M A C=1$. Similar behaviors of the evolution of these variables 


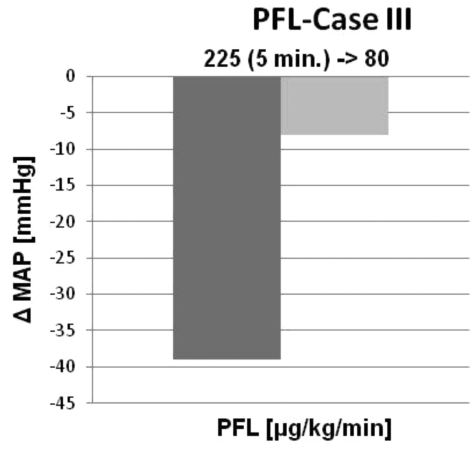

a. $M A P$

PFL-Case III

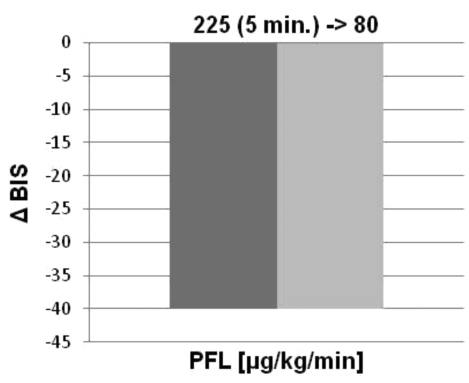

c. $B I S$

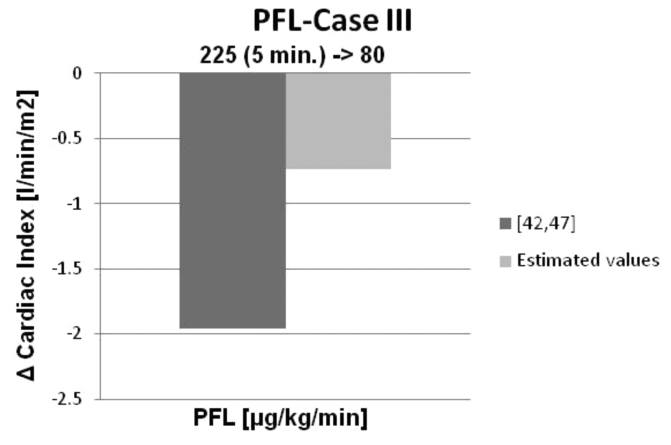

b. Cardiac index

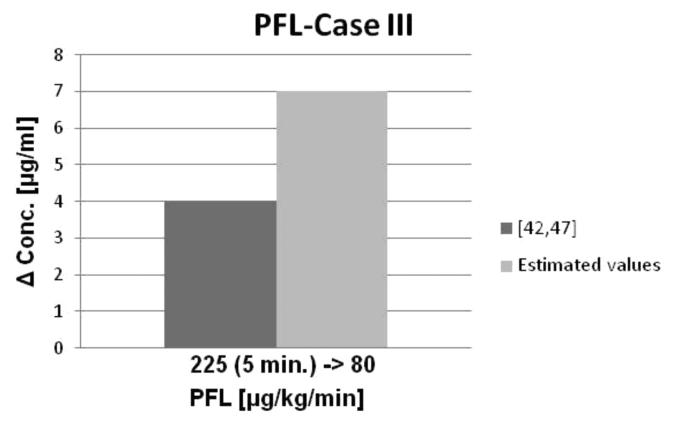

d. PFL Concentration

Figure 14. Case III: Difference between initial and final variables states predicted by the model (light bars) versus data reported in Larsen et al. ${ }^{42}$ and Mortier et al. ${ }^{46}$ (dark bars), when $225 \mu \mathrm{g} / \mathrm{kg} / \mathrm{min}$ of PFL is administrated for $5 \mathrm{~min}$, followed by an average infusion rate of $80 \mu \mathrm{g} / \mathrm{kg} / \mathrm{min}$ of the same drug. (a) MAP; (b) Cardiac Index; (c) BIS; (d) PFL concentration.

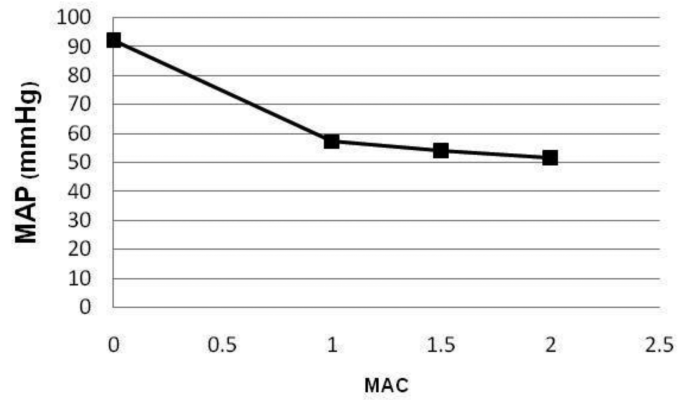

a. $M A P$

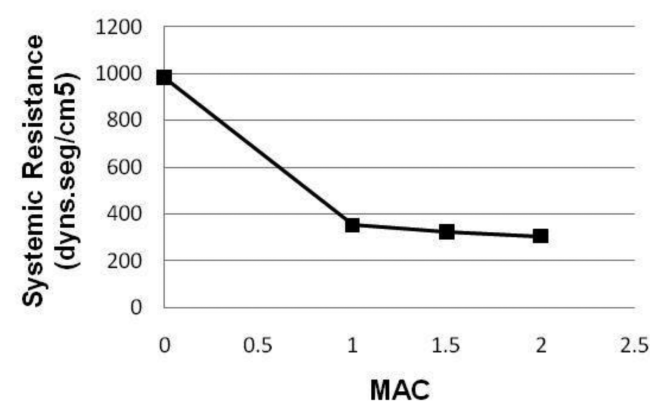

c. Systemic resistance

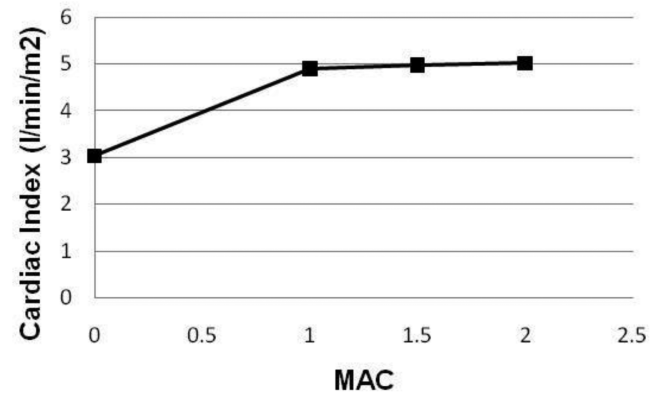

b. Cardiac index

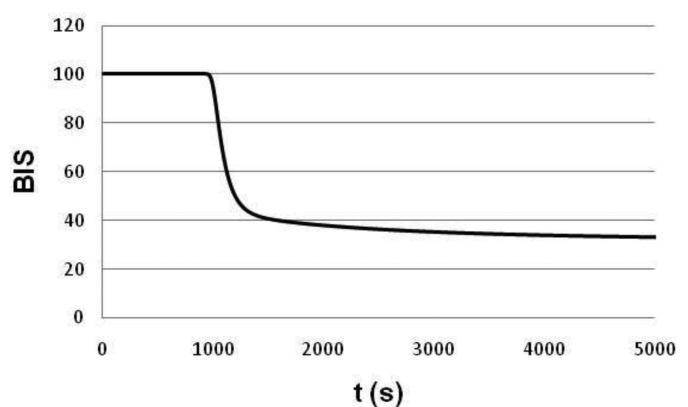

d. $B I S(M A C=1)$

Figure 15. Case IV: Variables profiles predicted by the model under the effects of an increasing $M A C$ value for isoflurane. (a) MAP; (b) Cardiac index; (c) Sistemic resistance; (d) BIS vs time.

with respect to $M A C$ or time are observed in Dua and Pistikopoulos ${ }^{17}$ and Malan et al. ${ }^{43}$
The comparison of the results for the infusion of ISO is shown in Figure 16. The variables compared against experimental data 


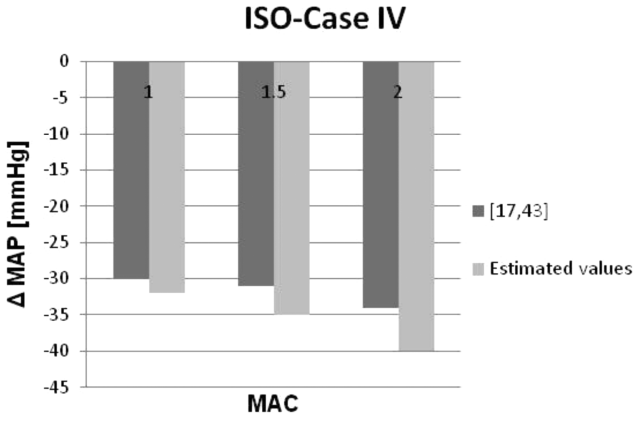

a. $M A P$

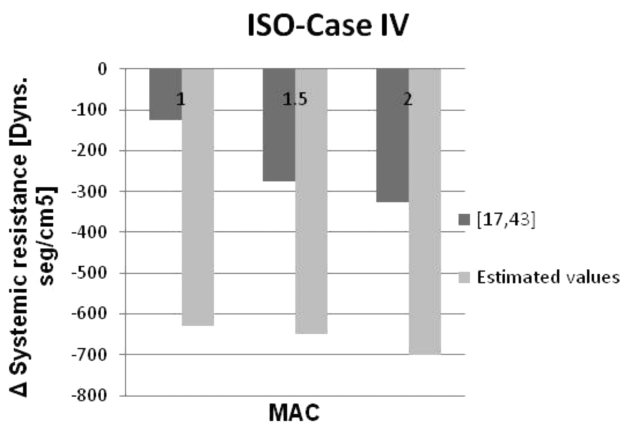

c. Systemic resistance

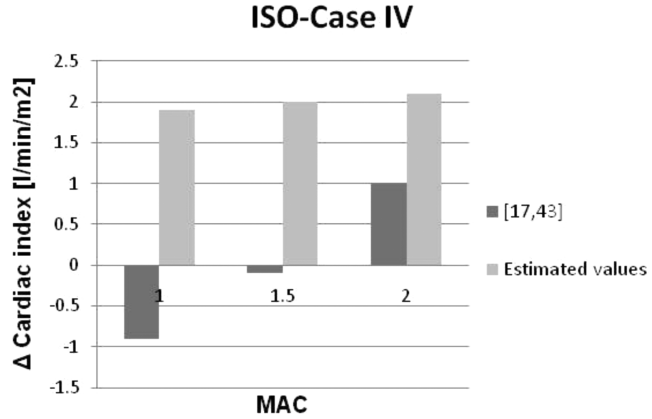

b. Cardiac index

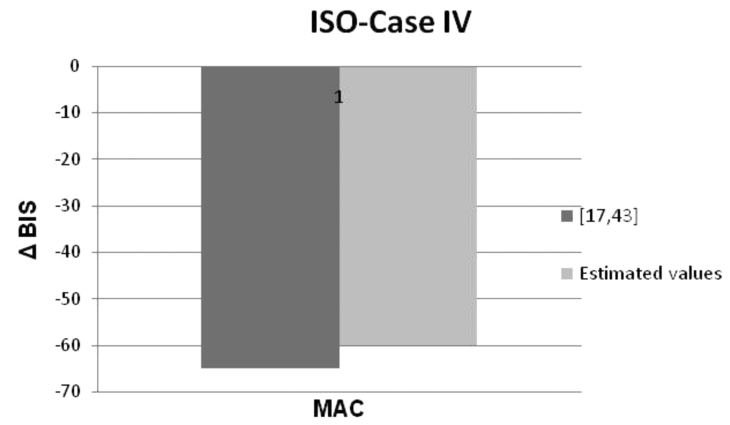

d. $B I S$

Figure 16. Case IV: Difference between initial and final variables states predicted by the model (light bars) versus data reported in Dua and Pistikopoulos ${ }^{17}$ and Malan et al. ${ }^{43}$ (dark bars): (a) MAP; (b) Cardiac Index; (c) Systemic resistance when ISO is administered with MAC 1, 1.5, and 2 and (d) BIS when ISO is administered with $M A C=1$.

taken from Dua and Pistikopoulos ${ }^{17}$ and Malan et al. ${ }^{43}$ are $M A P$, cardiac index, systemic resistance, and BIS.

The model correctly predicts the trend and magnitude of changes in MAP and BIS after the administration of ISO for the different $M A C$ values (Figure 16a and d, respectively). The systemic resistance decreases in agreement with the experience conducted in Malan et al., ${ }^{43}$ but the magnitude of the change is overestimated in all cases (Figure 16c). Finally, the response of the model for the cardiac index increases opposite to the experimental evidence for $M A C$ concentrations of 1 and 1.5 , nevertheless for $M A C=2$, the trend is correct.

Case V: Additional Studies with Dopamine (DP). In order to further analyze the behavior of the integrated physiological model to changes in the infusion profile of DP, additional results are provided in Figure 17. For this study, it was simulated the infusion of $2 \mu \mathrm{g} / \mathrm{kg} / \mathrm{min}$ of DP followed by increases of $2 \mu \mathrm{g} / \mathrm{kg} / \mathrm{min}$ every $30 \mathrm{~min}$ until $8 \mu \mathrm{g} / \mathrm{kg} / \mathrm{min}$ following the experiments reported in Leier et al. ${ }^{41}$ (white circles mark the occurrence of infusion changes).

Figure $17 \mathrm{a}$ and $\mathrm{b}$ show the effects of DP on the cardiac index, and on the net volume index of the left ventricle (net volume of left ventricle divided by body surface), respectively. It can be observed that in order to achieve a significant change in the cardiac index, the DP infusion must exceed $4 \mu \mathrm{g} / \mathrm{kg} / \mathrm{min}$. Increasing doses of DP from 6 to $8 \mu \mathrm{g} / \mathrm{kg} / \mathrm{min}$ do not produce a significant change on this index. A similar behavior is observed in the net volume index. ${ }^{41}$
In Figure $17 \mathrm{c}$, it can be seen that the systolic pressure increases significantly for the $4 \mu \mathrm{g} / \mathrm{kg} / \mathrm{min}$ dose. This trend is also evident to a lesser extent for diastolic pressure. In the case of the systemic resistance, decreases are observed for increasing doses of DP (Figure 17d). The most pronounced change also corresponds to the $4 \mu \mathrm{g} / \mathrm{kg} / \mathrm{min}$ infusion. Finally, heart rate increases with an increasing dose of DP (Figure 17e). The change is the greatest for the $4 \mu \mathrm{g} / \mathrm{kg} / \mathrm{min}$ dose.

The qualitative validation of the model to the administration of different doses of DP shows that the trends are correct for the variables analyzed, but the magnitude of change is not accurately predicted in general (Figure 18).

By inspecting the results for cases I to $\mathrm{V}$, it is observed that, in general, the model correctly predicts the trends of most studied variables in the front of the different drug infusions. Regarding magnitude, some variables were quite accurately predicted, whereas the rest verified significant discrepancies. This suggests that corrections should be introduced on parameter values and underlying relationships.

It should be stressed that the proposed model has a large number of parameters. For development and testing purposes, the adopted parametrization, taken from literature, corresponds to an "average" individual. However, it is well known that the response of the state variables to the different disturbances is patient specific and heavily dependent on attributes such as gender and age, among others. The observed discrepancies are mainly attributed to this lack of specificity of the model, which should be overcome by parameter estimation studies in order to generate patient specific parametrizations. 


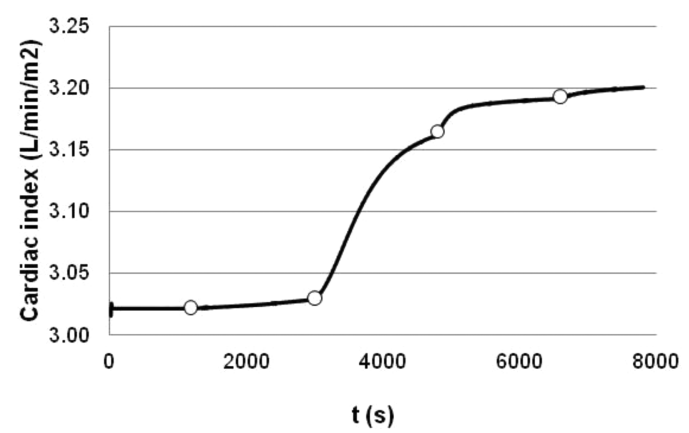

a. Cardiac index

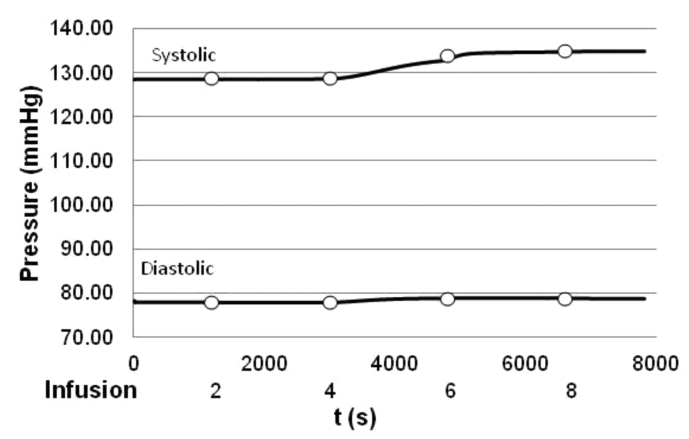

c. Systolic and diastolic pressures

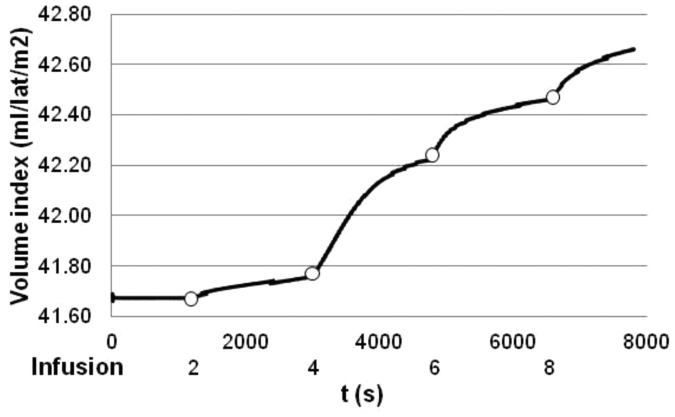

\section{b. Net Volume index of left ventricle}

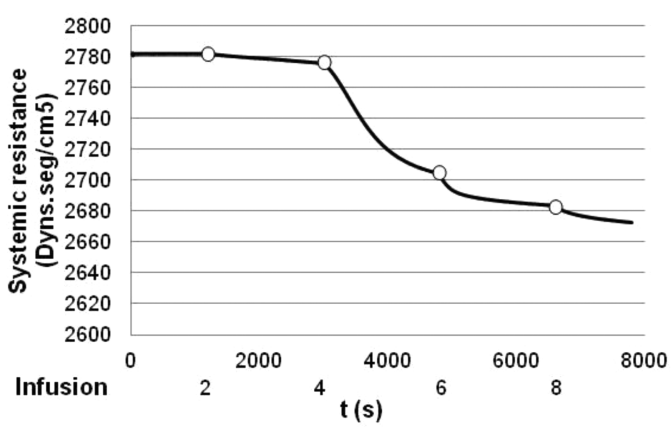

d. Systemic resistance

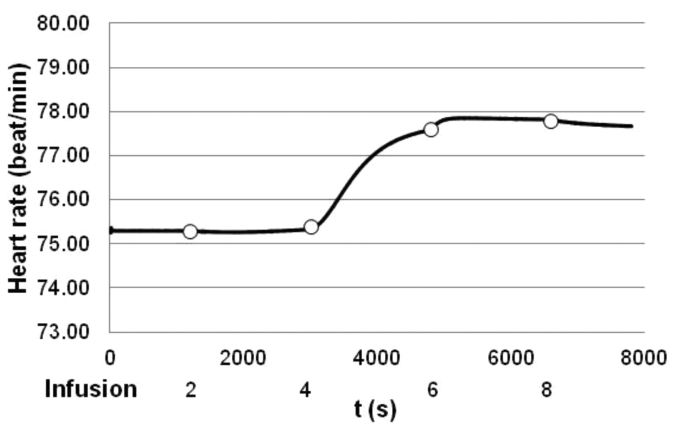

\section{e. Cardiac frequency}

Figure 17. Case V: Model variable time profile under the effects of a DP stepwise delivery. (a) Cardiac index; (b) net volume index of left ventricle; (c) systolic and diastolic pressures; (d) systemic resistance; (e) heart rate. Dots mark the infusion change.

\section{CONCLUSIONS AND FUTURE WORK}

This paper described the development and computational implementation of a cardiovascular-respiratory-pharmacodynamic model that integrates several state of the art lumped parameter physiological submodels taken from the open literature. The model was used to perform experiments using four different drugs to control mean arterial pressure $(M A P)$, cardiac output $(C O)$ and bispectral index $(B I S)$, also based on published studies. The simulation results suggest that the basic hemodynamic and pharmacodynamic processes of the human physiology under typical drug infusion scenarios can be reproduced in reasonable times with the described model.

Basically, it was verified that the correct trends of most variables can be reproduced, although quantitative validation of the complete model from clinical experiences is required to accurately predict the magnitude of the changes. This effort would demand the interaction of model developers with physicians and health institutions and counting with enough patient data gathered following current data collection protocols.

In particular, the integrated model has about of 110 parameters, which were held constant at standard mean values representative of a normal human individual. However, many parameters are patient specific. Consequently, to use the model as a diagnosis or decision making support tool, it is necessary to estimate such parameters in each case. This can be done with the same integrated model by feeding appropriate measurements obtained from the patient and running the model in parameter estimation mode.

Model behavior and computational performance observed during the simulation studies encourages the trial of the model in 


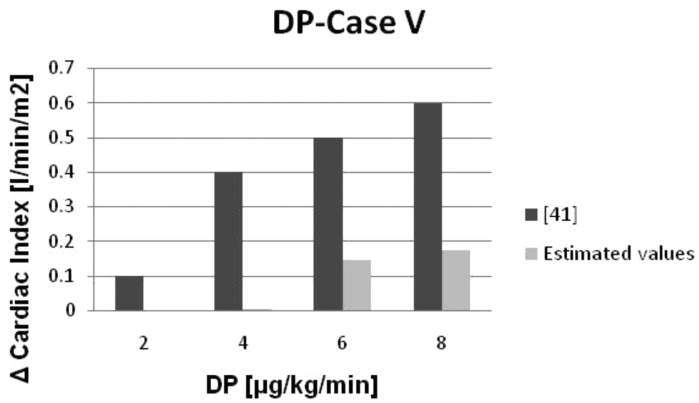

a. Cardiac Index

DP-Case V

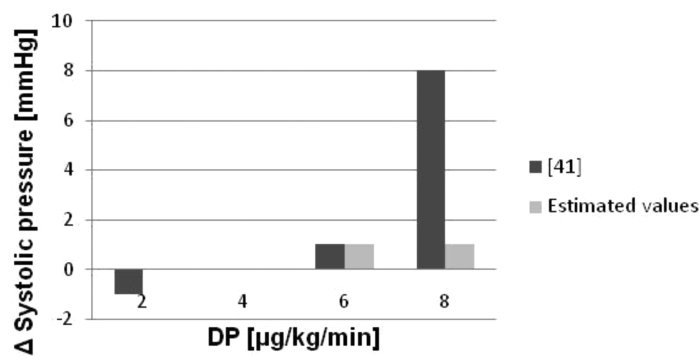

c. Systemic pressure

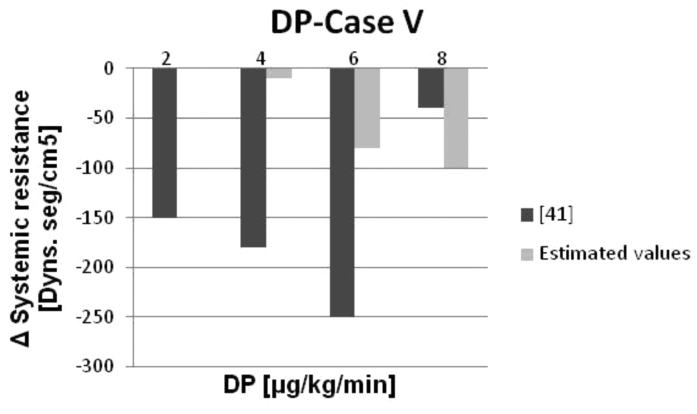

e. Systemic resistance

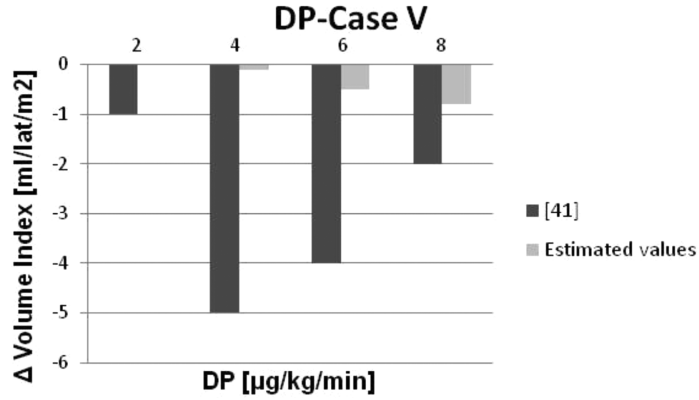

b. Volume index

DP-Case V

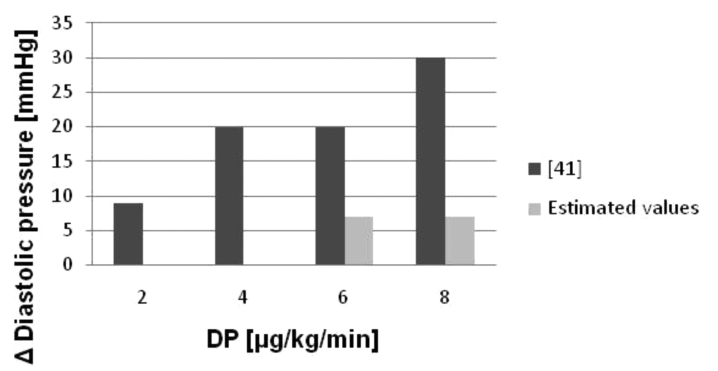

d. Diastolic pressure

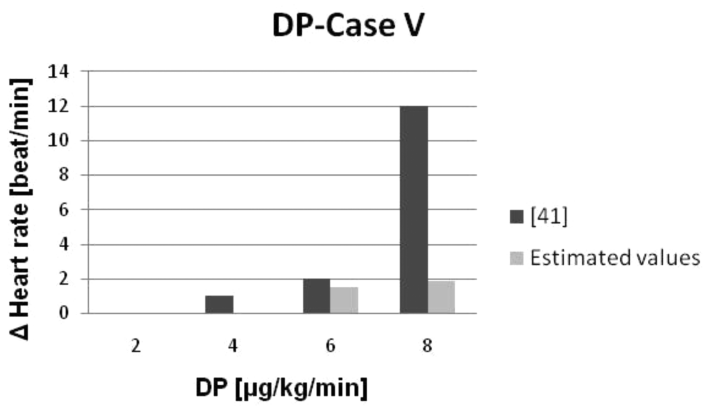

f. Heart rate

Figure 18. Case V: Difference between initial and final variable states predicted by the model (light bars) when infusions of DP of $2,4,6$, and $8 \mu \mathrm{g} / \mathrm{kg} /$ min are administered, and the values reported in Leier et al. ${ }^{41}$ (dark bars). (a) Cardiac index; (b) volume index; (c) systolic pressure; (d) diastolic pressure; (e) heart rate; (f) systemic resistance.

other type of studies for physiological investigation. For example, in parameter estimation mode, the model could be used to diagnose potential causes of dysfunction or disease by identifying the set of parameters that significantly shift from a healthy level when tuned, making use of patient data (measurements). Additionally, dynamic optimization studies can be carried out with the aim of identifying time profiles of the manipulated variables (drug infusion rates) in order to optimize sound, often conflicting, medical performance indicators. For example, it could be desirable to minimize the amount of administrated drug to achieve a specific target on a certain physiological variable while ensuring that other variables of interest fluctuate within safe bounds. In both cases (parameter estimation and dynamic optimization), the hybrid nature (continuous/discrete) of the model requires the adopted optimization engine to be able to deal with the inherent nondifferentiability.

\section{ASSOCIATED CONTENT}

S Supporting Information

Cardiovasular submodel equations, variables, and parameters; baroreflex submodel equations, variables, and parameters; respiratory submodel equations, variables, and parameters; gas transport and distribution submodel equations, variables, and parameters; and phycodynamic submodel equations, variables, and parameters. This material is available free of charge via the Internet at http://pubs.acs.org.

\section{AUTHOR INFORMATION}

\section{Corresponding Author}

*E-mail: abandoni@plapiqui.edu.ar. Tel.: +54 291 4861700. Fax: +542914861600.

Notes

The authors declare no competing financial interest. 


\section{ACKNOWLEDGMENTS}

This work was partially supported by Consejo Nacional de Investigaciones Cientificas y Técnicas, Agencia Nacional de Promoción Científica y Técnica and Universidad Nacional del Sur (Argentina).

\section{REFERENCES}

(1) Hunter, P. J.; Borg, T. K. Integration from proteins to organs: the Physiome Project. Nat. Rev. Mol. Cell Biol. 2003, 4 (3), 237-243.

(2) ITFoM-Information Technology Future of Medicine. Future and Emerging Technologies Flagship Initiatives. http://www.itfom.eu/ (accessed June 2014)

(3) Ottesen, J. T. The Mathematical Microscope-Making the inaccessible accessible. In BetaSys: Systems Biology of Regulated Exocytosis in Pancreatic $\beta$-Cells; Booß-Bavnbek, B., Klösgen, B., Larsen, J., Pociot, F., Renström, E., Eds.; Systems Biology, Volume 2; Springer: New York, 2011; Chapter 6, 97-121.

(4) Guyton, A.; Coleman, T. G.; Granger, H. J. Circulation: Overall regulation. Annu. Rev. Physiol. 1972, 34, 13-46.

(5) Ottesen, J.; Olufsen, M.; Larsen, J. Applied Mathematical Models in Human Physiology; SIAM: Philadelphia, 2004.

(6) Malpas, S. Editorial comment: Montani versus Osborn exchange of views. Exp. Physiol. 2009, 94 (4), 381-97.

(7) Korakianitis, T.; Shi, Y. A concentrated parameter model for the human cardiovascular system including heart valve dynamics and atrioventricular interaction. Med. Eng. Phys. 2006, 28, 613-28.

(8) Vallverdú Ferrer, M. Modelado y Simulación del Sistema Cardiovascular en Pacientes con Lesiones Coronarias. Ph.D. Thesis, Universitat Politecnica de Catalunya, 1993.

(9) Smith, B. Minimal Hemodynamic Modelling of the Heart \& Circulation for Clinical Application. Ph.D. Thesis, Canterbury University, New Zealand, 2003.

(10) Summers, R.; Ward, K.; Witten, T.; Convertino, V.; Ryan, K.; Coleman, T.; Hester, R. Validation of a computational platform for the analysis of physiologic mechanisms of a human experimental model of hemorrhage. Resuscitation 2009, 80, 1405-10.

(11) Hann, C.; Chase, J.; Desaive, T.; Froissart, C.; Revie, J.; Steveneson, D.; Lambermont, B.; Ghuysen, A.; Kolh, P.; Shaw, G. Unique parameter identification for model-based cardiac diagnosis in critical care. Proceedings of the 7th IFAC Symposium on Modelling and Control in Biomedical Systems; Aalborg: Denmark, 2009.

(12) Samar Z. Cardiovascular Parameter Estimation using a Computational Model. M.Sc. Thesis, Massachusetts Institute of Technology, 2005.

(13) Dua, P.; Pistikopulos, E. Modelling and control of drug delivery systems. Comput. Chem. Eng. 2005, 29, 2290-96.

(14) Gopinath, R.; Bequette, B.; Roy, R.; Kaufman, H. Issues in the design of a multirate model- based controller for a nonlinear drug infusion system. Biotechnol. Prog. 1995, 11 (3), 318-32.

(15) Rao, R.; Bequette, B.; Roy, R. Simultaneous regulation of hemodynamic and anesthetic states: a simulation study. Ann. Biomed. Eng. 2000, 28, 71-84.

(16) Gentilini, A.; Frei, C.; Glattfedler, A.; Morari, M.; Sierber, T.; Wymann, R; Schnider, T.; Zbinden, A. Multitasked closed-loop control in anesthesia. IEEE Eng. Med. Biol. 2001, 20, 29-53.

(17) Dua, P.; Pistikopoulos, E. Modelling and multi-parametric control for delivery of anaesthetic agents. Med. Biol. Eng. Comput. 2010, 48, 543-53.

(18) Sreenivas, Y.; Lakshminarayanan, S.; Rangaiah, G. P. Advanced Control Strategies for the Regulation of Hypnosis with Propofol. Ind. Eng. Chem. Res. 2009, 48 (8), 3880-3897.

(19) Sreenivas, Y.; Yeng, T. W.; Rangaiah, G. P.; Lakshminarayanan, S. Comprehensive Evaluation of PID, Cascade, Model-Predictive, and RTDA Controllers for Regulation of Hypnosis. Ind. Eng. Chem. Res. 2009, 48 (12), 5719-5730.

(20) Ursino, M.; Magosso, E.; Avanzolini, G. An integrated model of the human ventilatory control system: response to hypercapnia. Clin. Physiol. 2001, 21 (4), 447-64.
(21) Christiansen, T.; Dræby, C. Modelling the respiratory system. Technical report IMFUFA; Roskilde University: Denmark, 1996; Text No. 318.

(22) Siggaard-Andersen, O.; Wimberley, P.; Fogh-Andersen, N.; Gøthgen, I. Measured and derived quantities with modern $\mathrm{pH}$ and blood gas equipment: Calculation algorithms with 54 equations. Scan. J. Lab. Invest. 1988, 48, 7-15.

(23) Ionescu C.; De Keyser R.; Torrico B.; De Smet T.; Struys M.; Normey-Rico J. Robust predictive control strategy applied for propofol dosing using BIS as a controlled variable during anesthesia. IEEE Trans. Biomed. Eng., 2008, 55 (9).

(24) Rosow C.; Manberg P. Bispectral Index Monitoring. Anesthesiol. Clin. North Am. 2001, 19 (4).

(25) Myles, P.; Leslie, K.; McNeil, J.; Forbes, A.; Chan, M. Bispectral index monitoring to prevent awareness during anaesthesia: the B-Aware randomised controlled trial . Lancet 2004, 363.

(26) Sebel, P.; Bowles, S.; Chamoun, N. EEG Bispectrum Predicts Movement during Thiopental /Isoflurane Anesthesia. J. Clin. Monit. 1995, 11, 83-91.

(27) Ghouri, A.; Monk, T.; White, P. Electroencephalogram spectral edge frequency, lower esophageal contractility, and autonomic responsiveness during general anesthesia. J. Clin. Monit. 1993, 9, 176185.

(28) Kearse, L.; Manberg, P.; Chamoun, N.; deBors, F.; Zaslavsky, A. Bispectral Analysis of the Electroencephalogram Correlates with Patients Movements to Skin Incisions during Propofol/Nitrous Oxide Anesthesia. Anesthesiology 1994, 81, 1365-1370.

(29) Glass, P.; Bloom, M.; Kearse, L.; Rosow, C.; Sebel, P.; Manberg, P. Bispectral analysis measures sedation and memory effects of Propofol, Midazolam, Isoflurane and Alfentanil in healthy volunteers. Anesthesiology 1997, 86, 836-847.

(30) Doi, M.; Gajraj, R.; Mantzaridis, H.; Kenny, G. Relationship between calculated blood concentration of propofol and electrophysiological variables during emergence from anaesthesia: a comparison of bispectral index, spectral edge frequency, median frequency and auditory evoked potential index. Br. J. Anaesthesia 1997, 78, 180-184.

(31) Rampil, I. A primer for EEG signal processing analysis. Anesthesiology 1998, 89, 980-1002.

(32) Schwilden, H.; Stoeckel, H.; Schüttler, J. Closed-loop feedback control of Propofol anaesthesia by quantitative EEG analysis in humans. Br. J. Anaesth. 1989, 62, 290-296.

(33) Park, T.; Barton, P. State event location in differential-algebraic models. ACM Trans. Model. Comput. Simul. 1996, 6 (2), 137-165.

(34) Pantelides, C. SpeedUp - Recent advances in process simulation. Comput. Chem. Eng. 1988, 12 (7), 745-755.

(35) Gopal, V.; Biegler, L. T. Nonsmooth dynamic simulation with linear programming based methods. Comput. Chem. Eng. 1997, 21 (7), 675-689.

(36) Bahl, V.; Linnenger, A. Hybrid simulation of continuous-discrete systems. In European Symposium on Computer Aided Process Engineering, 10th ed.; Pierucci, S., Ed.; Elsevier: Amsterdam, 2000; pp 163-168.

(37) Mao, G.; Petzold, L. R. Efficient integration over discontinuities for differential-algebraic systems. Comput. Math. Appl. 2002, 43, 65-79.

(38) Olufsen, M.; Ottesen, J.; Tran, H.; Ellwein, L.; Lipsitz, L.; Novak, V. Blood pressure and blood flow variation during postural change from sitting to standing: model development and validation. J. Appl. Physiol. 2005, 99, 1523-37.

(39) Ashour, S. S.; Hanna, O. T. A New Very Simple Explicit Method for the Integration of Mildly Stiff Ordinary Differential Equations. Comput. Chem. Eng. 1990, 14 (3), 267-272.

(40) Hvala, N.; Strmčnik, S.; Šel, D.; Milanič, S.; Banko, B. Influence of model validation on proper selection of process models - an industrial case study. Comput. Chem. Eng. 2005, 29, 1507-1522.

(41) Leier, C.; Heban, P.; Huss, P.; Bush, C.; Lewis, R. Comparative systemic and regional hemodynamic effects of dopamine and dobutamine in patients with cardiomyopathic heart failure. Circulation 1978, $58,466-75$.

(42) Larsen, R.; Rathgeber, J.; Bagdhan, A.; Lange, H.; Rieke, H. Effects of propofol on cardiovascular dynamics and coronary blood flow in 
geriatric patients. A comparison with etomidate. Anaesthesia 1988, 43, $25-31$.

(43) Malan, T.; DiNardo, J.; Isner, R.; Frink, E.; Goldberg, M.; Fenster, P. Cardiovascular effects of Sevoflurane compared with those of Isoflurane in Volunteers. Anesthesiology 1995, 83, 918-28.

(44) MacGregor, D.; Smith, T.; Prielipp, R.; Butterworth, J.; James, R.; Scuderi, P. Pharmacokinetics of Dopamine in Healthy Male Subjects. Anesthesiology 2000, 92, 338-46.

(45) Hughes, M.; Glass, P.; Jacobs, J. Context-sensitive Half-time in Multicompartment Pharmacokinetic Models for Intravenous Anesthetic Drugs. Anesthesiology 1992, 76, 334-341.

(46) Mortier, E.; Struys, M.; De Smet, T.; Versichelen, L.; Rolly, G. Closed-loop controlled administration of propofol using bispectral analysis. Anaesthesia 1998, 53, 749-754.

(47) Yasuda, N.; Lockhart, S.; Eger, E., II; Weiskopf, R.; Johnson, B.; Freire, B.; Fassoulaki, A. Kinetics of Desflurane, Isoflurane, and Halothane in Humans. Anesthesiology 1991, 74, 489-498. 